\title{
Erratum: Determination of multicritical points for lattice-gas models by finite-size scaling of the susceptibility [Phys. Rev. B 32, 4756 (1985)]
}

\author{
Per Arne Rikvold*
}

The asymptotic scaling relation for the maximum susceptibility that was given in the above paper applies to the special case of a vanishing density-density correlation-function exponent $\eta$. The general form for a $d$-dimensional strip of cross section $N^{d-1}$ is $x N^{\max } \sim N^{d-1} \hat{\xi}_{N^{-d-\eta}}$ as $N \rightarrow \infty$. Thus, the leading term in the second line of Eq. (7) should be of the order of $N^{1-\eta_{m}}$, rather than $N$, and the leading term in the second line of Eq. (10) should be of order $N^{-\left(1+\eta_{m}\right)}$. Here $\eta_{m}$ is the multicritical value of $\eta$. From the standard scaling form for the free energy per site one obtains $x_{N}^{\max } \sim N^{2 y_{T}-d}$. Here $y_{T}$ is the thermal eigenvalue. Thus, the appropriate $\eta_{m}$ is $\eta_{m}=2-2 y_{T}+d$, which is the correlation-function exponent corresponding to an approach to the multicritical point parallel to the line of critical points. For two-dimensional Ising models (which includes the simple lattice-gas model considered in the paper) the multicritical value of $y_{T}$ is $\frac{9}{5},{ }^{2}$ so that $\eta_{m}=\frac{2}{5}$ in this case.

The factor $A_{2}$ in the third lines of Eqs. (7) and (10), which describes the behavior of $\chi_{N}^{\max }$ at a second-order transition, may depend logarithmically on $N$, due to coupling to the specific heat. For non-Ising-like systems its dependence on $N$ might be stronger.

Although conceptually significant, because of the strong exponential divergence below the multicritical temperature these corrections to the scaling relations are numerically unimportant for the system sizes considered. In the paper the effects of unknown corrections to scaling were taken into account, and the numerical results obtained remain valid within the given accuracy.

I am grateful to M. E. Fisher, M. P. Nightingale, and T. L. Einstein for pointing out these inaccuracies in the scaling relations.

"Permanent address: ChemLink Industrial/Petroleum Chemicals
Division, ARCO Chemical Company, 3801 West Chester Pike,
Newtown Square, PA 19073.

${ }^{1}$ I. D. Lawrie and S. Sarbach, in Phase Transitions and Critical
Phenomena, edited by C. Domb and J. L. Lebowitz (Academic, London, 1984), Vol. 9; R. B. Griffiths, Phys. Rev. B 7, 545 (1973).

${ }^{2}$ D. A. Huse, Phys. Rev. Lett. 49, 1121 (1982).

\section{Erratum: Wetting of a glass substrate by a binary liquid mixture [Phys. Rev. B 33, 402 (1986)]}

Xiao-lun Wu, Mark Schlossman, and Carl Franck

We would like to note the following corrections to our paper:

(1) Reference 1 has the word "Faraday" misspelled. Faraday is the correct spelling.

(2) Reference $4(\mathrm{~g})$ should read, B. M. Law (unpublished). The reference given is not correct as it stands. 